\title{
Coding of Sounds in the Auditory System and Its Relevance to Signal Processing and Coding in Cochlear Implants
}

\author{
Brian C. J. Moore \\ Department of Experimental Psychology, University of Cambridge, Downing Street, \\ Cambridge CB2 3EB, England
}

\begin{abstract}
Objective: To review how the properties of sounds are "coded" in the normal auditory system and to discuss the extent to which cochlear implants can and do represent these codes. Data Sources: Data are taken from published studies of the response of the cochlea and auditory nerve to simple and complex stimuli, in both the normal and the electrically stimulated ear. Review Content: The review describes: 1) the coding in the normal auditory system of overall level (which partly determines perceived loudness), spectral shape (which partly determines perceived timbre and the identity of speech sounds), periodicity (which partly determines pitch), and sound location; 2) the role of the active mechanism in the cochlea, and particularly the fast-acting compression associated with that mechanism; 3) the neural response patterns evoked by cochlear implants; and 4) how the response patterns evoked by implants differ from those observed in the normal auditory system in
\end{abstract}

response to sound. A series of specific issues is then discussed, including: 1) how to compensate for the loss of cochlear compression; 2) the effective number of independent channels in a normal ear and in cochlear implantees; 3 ) the importance of independence of responses across neurons; 4) the stochastic nature of normal neural responses; 5) the possible role of across-channel coincidence detection; and 6) potential benefits of binaural implantation.

Conclusions: Current cochlear implants do not adequately reproduce several aspects of the neural coding of sound in the normal auditory system. Improved electrode arrays and coding systems may lead to improved coding and, it is hoped, to better performance. Key Words: Binaural hearing-Cochlear implants-Loudness-Neural coding-Pitch-Stochastic neural responses-Timbre.

Otol Neurotol 24:243-254, 2003.
The properties of sounds are represented in the normal auditory system in the spatial and temporal patterns of nerve spikes in the auditory nerve and higher centers of the auditory pathway. There is still controversy about exactly what aspects of the spatial and temporal patterns represent "codes" for particular stimulus properties. To be a code, a specific aspect of the neural response pattern should be used by the brain to determine one or more properties of a stimulus, and changes in that aspect of the response pattern should affect the perception of the stimulus. In this review, I shall describe aspects of neural responses to sound that have been proposed to act as codes, and I shall describe the extent to which cochlear implants reproduce the appropriate patterns of neural response. Although most readers will be familiar with the basic anatomy and physiology of the auditory system, I

Supported by the Medical Research Council (U.K.), Royal National Institute for Deaf People (U.K.), and Defeating Deafness (U.K.).

Address correspondence and reprint requests to Brian C. J. Moore, Ph.D., Department of Experimental Psychology, University of Cambridge, Downing Street, Cambridge CB2 3EB, UK; Email: bcjm@cus.cam.ac.uk start with a brief review of these topics, because this will help in understanding how the various codes work. It is assumed that the reader has some basic familiarity with cochlear implants and how they work. For an introduction to this topic see Parkins (1).

The cochlea is divided along its length by two membranes: Reissner's membrane and the basilar membrane (BM). Inward movement of the oval window, produced by movement of the stapes, results in a corresponding outward movement in a membrane covering a second opening in the cochlea: the round window. Such movements result in pressure differences between one side of the BM and the other (i.e., the pressure differences are applied in a direction perpendicular to the BM), and this results in movement of the BM.

A third membrane, the tectorial membrane, lies close to and above the BM and also runs along the length of the cochlea. Between the BM and the tectorial membrane are hair cells, which form part of the organ of Corti (Fig. 1). The hair cells are divided into two groups by an arch known as the tunnel of Corti. Those on the side of the arch closest to the outside of the spiral shape are known as outer hair cells (OHCs), and they are arranged in three 


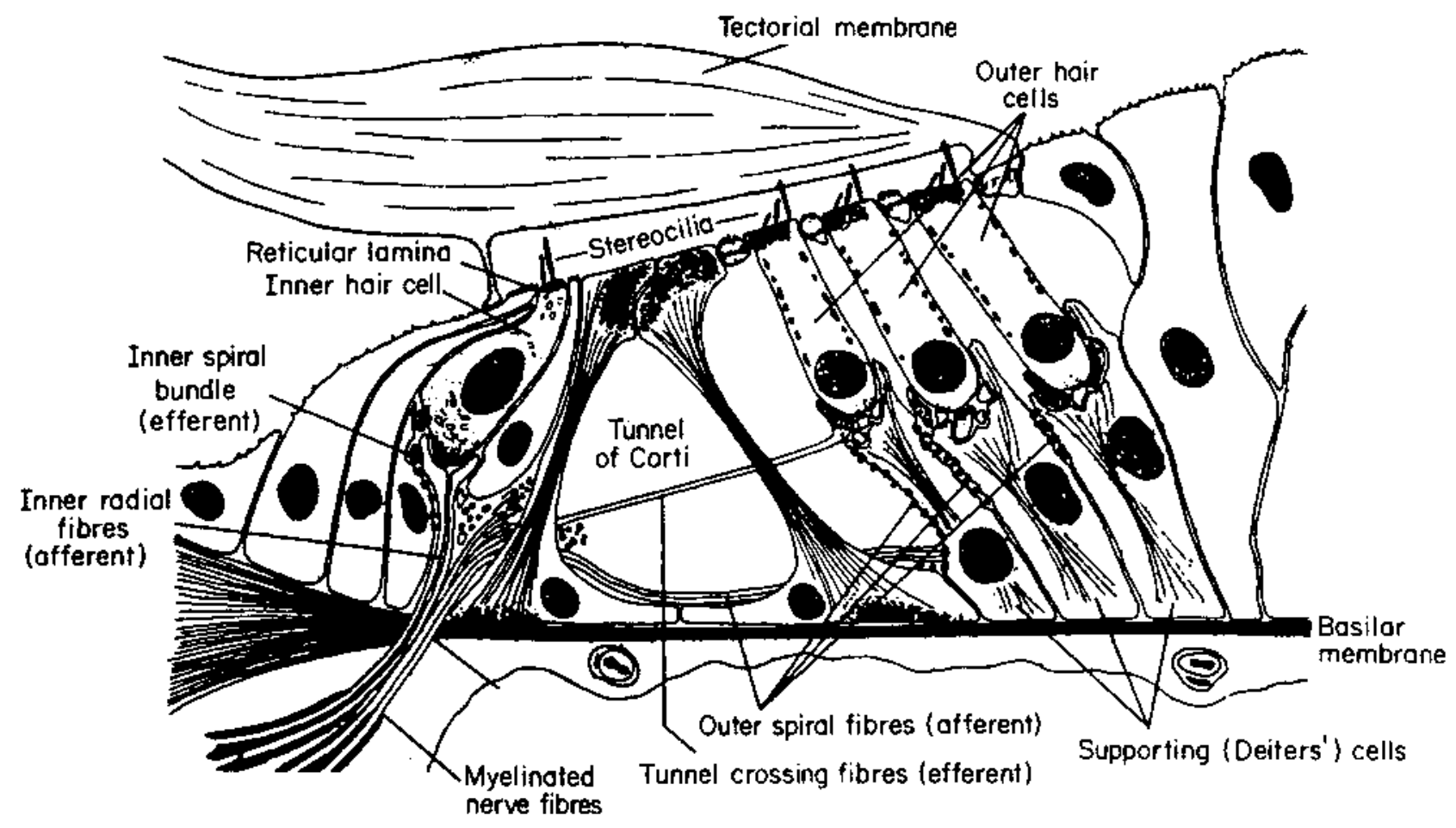

FIG. 1. Cross-section of the organ of Corti.

to five rows. The hair cells on the other side of the arch form a single row and are known as inner hair cells (IHCs). The stereocilia of the OHCs touch the tectorial membrane, but this may not be true for the IHCs. The tectorial membrane is effectively hinged at one side (off to the left in Fig. 1). When the BM moves up and down, a shearing motion is created; the tectorial membrane moves sideways (in the left-right direction in Fig. 1) relative to the tops of the hair cells. As a result, the stereocilia at the tops of the hair cells are moved sideways. The deflection of the stereocilia of the IHCs leads to a flow of electric current through the IHCs, which in turn leads to the generation of action potentials (nerve spikes) in the neurons of the auditory nerve. Thus, the IHCs act to convert mechanical movements into neural activity. In a profoundly hearing-impaired person, who would be a candidate for a cochlear implant, the IHCs are usually severely damaged or missing altogether, and the neurons that make up the auditory nerve may also be partially degenerated $(2,3)$.

The main role of the OHCs is probably to actively influence the mechanics of the cochlea. The OHCs have a motor function, changing their length, shape, and stiffness in response to electric stimulation (4-6), and they can therefore influence the response of the BM to sound. The OHCs are often described as being a key element in an active mechanism within the cochlea. The exact way in which the active mechanism works is complex and is still not fully understood. For recent reviews, the reader is referred to (6-9).

The response of the BM to stimulation with a sine- wave (also called a sinusoid or pure tone) takes the form of a wave that moves along the BM from the base toward the apex (10). The amplitude of the wave increases at first with increasing distance from the base and then decreases rather abruptly. The basic form of the wave is illustrated in Figure 2, which shows schematically the

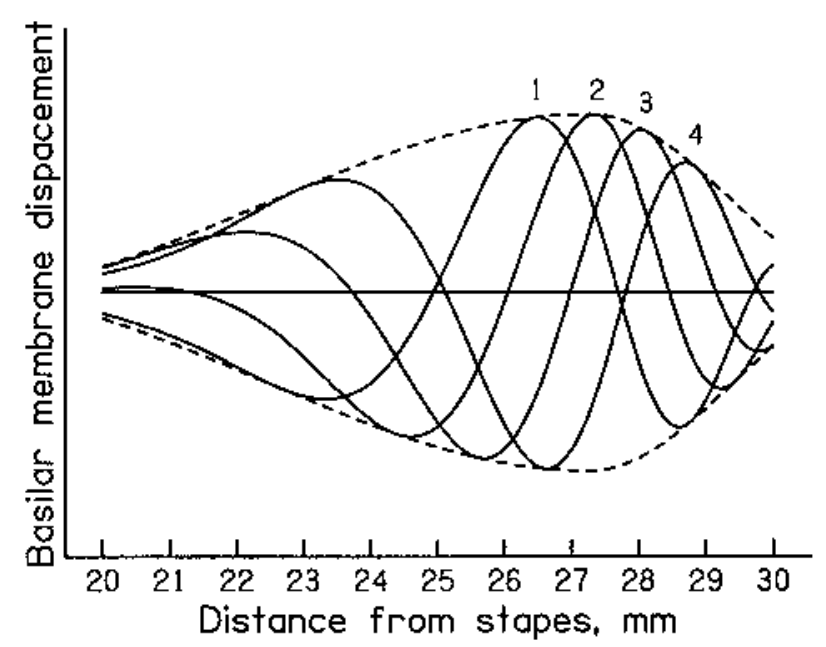

FIG. 2. Schematic illustration of the instantaneous displacement of the basilar membrane for four successive instants in time, in response to a low-frequency sinewave. The four successive peaks in the wave are labeled 1,2, 3, and 4. Also shown is the line joining the amplitude peaks, which is called the envelope. The response shown here is typical of what would be observed in a dead ear. 
instantaneous displacement of the BM for four successive instants in time, in response to a low-frequency sinewave. Figure 2 also shows the line joining the amplitude peaks, which is called the envelope. The envelope shows a peak at a particular position on the BM.

The response of the BM to sounds of different frequencies is strongly affected by its mechanical properties, which vary progressively from base to apex. At the base, the BM is relatively narrow and stiff. This causes the base to respond best to high frequencies. At the apex, the BM is wider and much less stiff, which causes the apex to respond best to low frequencies. Each point on the BM is tuned; it responds best (with greatest displacement) to a certain frequency-the characteristic frequency $(\mathrm{CF})$, or best frequency - and responds progressively less as the frequency is moved away from the CF. The CF decreases monotonically with distance from the base. It is now believed that the tuning of the BM arises from two mechanisms. One is referred to as the passive system or passive mechanism. This depends on the mechanical properties of the BM and surrounding structures, and it operates in a roughly linear way. The other mechanism is the active mechanism. This depends on the operation of the OHCs, and it operates in a nonlinear way. The active mechanism depends on the cochlea being in good physiological condition, and it is easily damaged. When the OHCs operate normally, the BM shows sharp tuning, especially for low input sound levels. The traveling wave illustrated in Figure 2 is representative of what might be observed in a dead cochlea, when only the passive system is operating. In a living healthy cochlea, the envelope of the traveling wave would have a much sharper peak.

A second function of the active mechanism is to provide level-dependent amplification (also called gain) on the BM. The gain is greatest for low-level inputs (levels below approximately $30 \mathrm{~dB}$ sound pressure level [SPL]), and it decreases progressively with increasing level for levels up to 90 to $100 \mathrm{~dB}$ SPL (11-13). This leveldependent gain means that the response on the $\mathrm{BM}$ is compressive. For example, if the input level of a sinewave is increased from 50 to $60 \mathrm{~dB}$ SPL, the response on the $\mathrm{BM}$ at the place tuned to the frequency of that sinewave may increase only by approximately $2.5 \mathrm{~dB}$. The compression occurs only for tones with frequencies that are reasonably close to the $\mathrm{CF}$ for the place whose response is being measured. Figure 3 is a schematic illustration of input-output functions on the BM.

\section{CODING OF SOUND LEVEL}

Coding in the normal auditory system

Loudness is a subjective attribute of sound and is related to an objective measure, the physical level; for sound levels above approximately $40 \mathrm{~dB}$ SPL, the loudness roughly doubles for each $10-\mathrm{dB}$ increase in sound level (14-16). It is commonly believed that sound level is coded in terms of neural firing rate. Loudness may be related to the total spike rate evoked by a sound, although

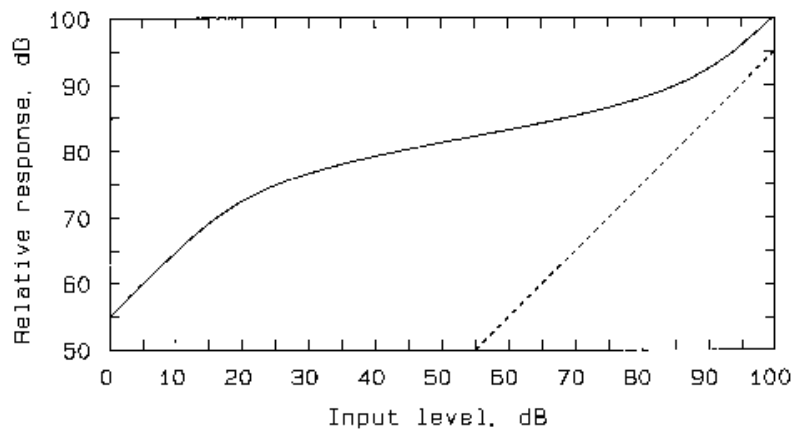

FIG. 3. Schematic illustration of input-output functions on the basilar membrane. The solid line shows a typical function in a normal ear for a sinewave input with frequency close to the characteristic frequency. The dashed line shows the function that would be observed in an ear in which the active mechanism was not operating.

there is some evidence that this is not quite correct (17). Figure 4 shows schematically how the rate of discharge for three auditory nerve fibers changes as a function of stimulus level. The curves are called rate-versus-level functions. In each case, the stimulus was a sinewave at the CF of the neuron. Consider first the curve labeled $(a)$. This curve is typical of what is observed for neurons with high spontaneous firing rates, which make up approximately $61 \%$ of the auditory nerve (18). Above a certain sound level, the neuron no longer responds to increases in sound level with an increase in firing rate; the neuron is said to be saturated. The range of sound levels between

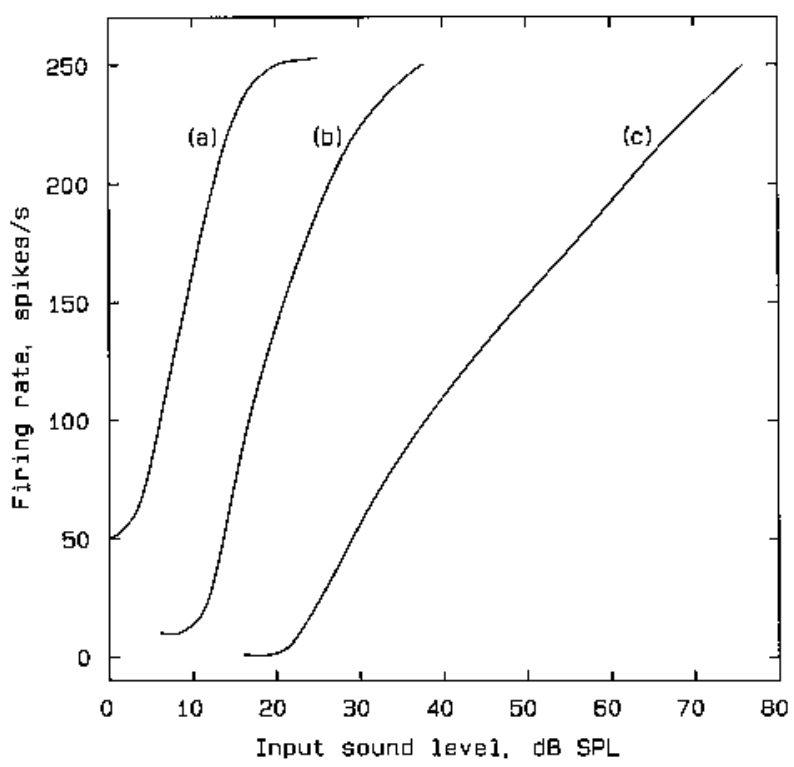

FIG. 4. Schematic examples of how the discharge rates of single auditory neurons vary as a function of stimulus level. The curves are called rate-versus-level functions. In each case, the stimulus was a sinewave at the characteristic frequency of the neuron. Curves $(a),(b)$, and $(c)$ are typical of what is observed for neurons with high, medium, and low spontaneous firing rates, respectively. 
threshold and the level at which saturation occurs is called the dynamic range. For neurons with high spontaneous rates, this range is often quite small, approximately 15 to $30 \mathrm{~dB}$. Curve $(b)$ is typical of what is observed for neurons with medium spontaneous rates, which make up approximately $23 \%$ of the auditory nerve (18). The threshold is slightly higher than for $(a)$, and the dynamic range is slightly wider. Curve $(c)$ is typical of what is observed for neurons with low spontaneous rates, which make up approximately $16 \%$ of the auditory nerve (18). The threshold is higher than for $(b)$. At first, the firing rate increases fairly rapidly with increasing sound level, but then the rate of increase slows down. The firing rate continues to increase gradually with increasing sound level over a wide range of levels. This has been called sloping saturation (19).

The variation of rate-versus-level functions across neurons depends mainly on the type of synapse with the IHC. Neurons with low thresholds have large sensitive synapses. They start to respond at very low sound levels, where the input-output function on the BM is nearly linear (Fig. 3). As the sound level increases, the BM displacement increases in a nearly linear manner, and the neuron saturates relatively early, giving a small dynamic range. Neurons with higher thresholds have less sensitive synapses. They respond over the range of sound levels where the BM input-output function shows a strong compressive nonlinearity (Fig. 3). Hence, a large increase in sound level is needed to increase the BM displacement to the point where the neuron saturates, and the neuron has a wide dynamic range.

If the level of sounds is coded in terms of neural firing rate, then, at high sound levels, the neurons with low spontaneous rates and wide dynamic ranges must play a crucial role $(20,21)$. The wide dynamic range of these neurons depends critically on the compression that occurs on the BM, which in turn depends upon the operation of the OHCs. I shall return to this point later.

Information about the level of sounds, and especially the relative levels of the different frequency components in complex sounds (such as speech) is also carried in the detailed time pattern of nerve spikes. In response to a sinewave, nerve spikes tend to be phase locked or synchronized to the stimulating waveform. A given nerve fiber does not necessarily fire on every cycle of the stimulus, but, when spikes do occur, they occur at roughly the same phase of the waveform each time. Thus, the time intervals between spikes are (approximately) integer multiples of the period of the stimulating waveform. For example, a 500-Hz sinewave has a period of 2 milliseconds, and the intervals between nerve spikes would be close to 2, 4, 6, and 8 milliseconds, and so on. In general, a neuron does not fire in a completely regular manner, so there are not exactly 500 , or 250 , or 125 spikes per second. However, information about the period of the stimulating waveform is carried unambiguously in the temporal pattern of firing. Phase locking occurs for frequencies up to approximately 4 to $5 \mathrm{kHz}$ but is lost above that (22).
Phase locking may be particularly important for coding the relative levels of components in complex sounds (23). When the level of a component is increased relative to that of the other components, the degree of phase locking to that component increases. In general, any change in the spectral composition of a complex sound results in a change in the pattern of phase locking as a function of $\mathrm{CF}$, provided the spectral change is in the frequency region below 4 to $5 \mathrm{kHz}$.

Heinz et al. (24) have proposed a way in which information from the timing of nerve spikes may be used to code level. They observed that different places on the $\mathrm{BM}$ vibrate with different phases. For example, when one point is moving upward, a nearby point may be moving downward, as can be seen from Figure 2. In response to a single sinewave, the nerve spikes evoked at one place on the BM will occur at different times from those evoked at an adjacent place. The phase response of the BM varies with sound level, and this level-dependent phase effect depends on the active mechanism in the cochlea. Heinz et al. (24) proposed that level-dependent changes in the phase response of the BM provide a code for level. The phase changes may be detected by the pattern of responses across an array of neurons, each of which receives inputs from two auditory nerve fibers with slightly different CFs and fires when the inputs from the two fibers are synchronous. Such neurons are called across-frequency coincidence detectors. It is assumed that the conduction time from the instant of spike initiation to the coincidence detector is different for the two "input" neurons and that this difference varies across coincidence detectors. In response to a tone of fixed frequency, the coincidence detector that responds most strongly would vary with sound level, and this may be (part of) the code for level.

\section{CODING IN COCHLEAR IMPLANTS}

In cochlear implants, level is typically coded by pulse magnitude (current), by pulse duration, or by analog current. Increases in any of these quantities lead to increased neural spike rates in the auditory nerve and hence to increasing loudness. With electric stimulation, the rate of increase of spike rate with increasing current (or pulse duration) is very rapid $(25,26)$ because (1) the compression on the BM, which depends on the operation of the active mechanism, is bypassed, and (2) neurotransmitter release is not required, so refractory effects play little role. Indeed, in response to electric stimulation, spike rates in single neurons can be greater than the maximum rates evoked by acoustic stimulation.

Consistent with the physiological observations of rapid increases in spike rate with increasing stimulating current, small changes in current or pulse width lead to large changes in loudness for human implantees. The range of currents between the detection threshold and the point at which an uncomfortable sensation occurs can be very small and is typically in the range 3 to $20 \mathrm{~dB}$ $(27,28)$. This is much less than the dynamic range of 
acoustic hearing, which is approximately $120 \mathrm{~dB}$ (16). In everyday life, a very large range of sound levels is encountered $(29,30)$. Hence, some form of compression is essential to map the wide range of input levels into the small usable range of currents in a cochlear implant.

In practice, compression is applied in implants in two stages. Many implants have an automatic gain control system at the "front end" that compresses the whole signal before it is filtered into several frequency bands. The filtered signals are then processed further. In some implant systems, each bandpass filtered signal is fed directly to one electrode (compressed analog stimulation). In other systems, the filtered signals are used to modulate rapid trains of biphasic current pulses (continuous interleaved sampling, CIS). Sometimes, a subset of signals is selected for delivery to a subset of electrodes. In all cases, further compression may be applied either to the analog signal, or in the mapping from the magnitude of the analog signal to the pulse width or height.

Compression systems vary in the speed with which they react to changes in the magnitude of their input. In acoustic hearing aids, where compression is used to compensate for the effects of loudness recruitment (31), fast compressors are often called syllabic compressors, because their gain changes over durations comparable to those of syllables (32-34). At the other extreme, very slow-acting systems are used to compensate for variations in overall sound level from one listening situation to another; these are often called automatic volume control systems (34-36). Both types of systems can be found in commercial hearing aids.

The front-end compressors used in cochlear implants vary considerably across different models in the speed of their response, although many can be described as medium speed compressors, somewhere between syllabic compressors and automatic volume control systems (37). Some implant systems offer compressors with different speeds as options in different programs.

The second stage of compression used in cochlear implants, applied in the transformation of the magnitude of the analog signal to the pulse width or height, is often instantaneous. In theory, fast or instantaneous compression would mimic the normal action of the cochlea, because the compression observed on the BM appears to act almost instantaneously (38). However, in practice, problems arise when fast or instantaneous compression is used as the sole means of compression.

Assume that it is desired to make sounds audible, but not uncomfortably loud, over a range of sound levels from approximately $30 \mathrm{~dB}$ to $120 \mathrm{~dB}$ SPL. To compress this range of $90 \mathrm{~dB}$ into the typical dynamic range of currents at the input to a single electrode (approximately $10 \mathrm{~dB}$ ) requires 9:1 compression. This is a very high compression ratio. It is known that if such a high compression ratio were used in a hearing aid with fast compression, it would result in a severe reduction in speech intelligibility $(39,40)$. Fast compression has deleterious effects for several reasons. First, important information in speech is carried in the patterns of amplitude modu- lation in different frequency bands $(41,42)$. Fast compression reduces the modulation depth (43), and this adversely affects speech intelligibility when the compression ratio is greater than approximately 2 , at least for normally hearing listeners $(44,45)$. Second, if fast compression is used before the signal is split into different frequency bands, this can introduce spurious amplitude fluctuations, which are correlated across different frequency bands. The correlated fluctuations can cause perceptual fusion of all bands (46), making it hard to separate target speech from background sounds. Finally, in implant systems using compressed analog stimulation, if fast compression is applied to the analog signal in each frequency band, this may distort the modulation pattern; for example "overshoot" and "undershoot" effects may occur. For all these reasons, it seems unwise to use fast or instantaneous compression as the sole means of compression.

A possible solution is to use a slow-acting front-end compressor to compensate for variations in overall sound level from one situation to another. The compressor can be designed with a supplementary "fast" component that prevents brief intense sounds from becoming uncomfortably loud (34-36). A slow compressor of this type has no effect on the amplitude modulation patterns that convey information in speech. When a slow front-end compressor is used, only a moderate amount of fast-acting compression is needed in the individual channels of an implant system. This use of slow compression preserves the amplitude modulation patterns, and it avoids spurious correlation of amplitude modulation patterns across channels, which would be expected to lead to an enhanced ability to separate speech from background sounds.

Finally, it is worth noting that no current implant system, to my knowledge, reproduces the effects of the level-dependent differences in phase response at different places on the BM. If these differences in phase response are used for the coding of sound level in a normal ear (24), then this code is entirely missing in implant systems.

\section{CODING OF SPECTRAL SHAPE}

\section{Coding in normal hearing}

The perceived quality or timbre of complex sounds is determined partly by their spectral shape. For example, each vowel sound has a spectrum with peaks at specific frequencies, called formant frequencies, and the patterning of the formant frequencies plays a large role in determining vowel quality and vowel identity. The perception of timbre in a normal ear depends on the frequency analysis performed in the cochlea. Each point on the BM behaves like a bandpass filter, responding most strongly to a limited range of frequencies; this range is referred to as the bandwidth. The BM as a whole behaves like an array of bandpass filters with center frequencies ranging from approximately $50 \mathrm{~Hz}$ to $15,000 \mathrm{~Hz}$. The bandwidth is roughly $13 \%$ of the center frequency, for center frequencies above approximately $1000 \mathrm{~Hz}$ (47). Filters with closely spaced center frequencies, say $1,000 \mathrm{~Hz}$ and 
$1,010 \mathrm{~Hz}$, would show very similar responses and thus cannot be regarded as conveying independent information. However, if the filter spacing is greater than the bandwidth of the filters, the filters would show relatively independent responses to a complex sound. In effect, there are approximately 39 independent filters. Within the frequency range important for speech perception (48) there are approximately 28 independent filters.

Spectral shape is represented by the relative response across filters, i.e., the magnitude of the response as a function of the center frequency, which is sometimes called the excitation pattern $(16,49)$. The relative level at the output of each filter may be coded in at least three ways:

1. By the relative firing rates of neurons as a function of CF (50). This is a "place" code for spectral shape.

2. By the relative amount of phase locking to the different frequency components in neurons with different CFs (23). For example, if a speech sound has a formant with a frequency of $1,400 \mathrm{~Hz}$, neurons with CFs around $1,400 \mathrm{~Hz}$ will show phase locking to the formant frequency.

3. By across-frequency coincidence detection of the type described earlier for the coding of sound level. For each spectral shape there will be a unique pattern at the output of the array of coincidence detectors.

\section{Coding in implants}

Within an implant processor, spectral shape is coded by filtering the signal into several frequency bands, as described above, and then mapping the filtered signals onto appropriate electrodes. This strategy is based on "place" coding in the normal auditory system-the first of the coding mechanisms described in the preceding paragraph. In an implant system, the effective number of independent bands, or channels, is limited by the number of electrodes and by the relative lack of isolation between electrodes. The effective number of channels is less than in a normal ear, and is typically less than eight, although the effective number may be somewhat greater with recently developed modiolar-hugging electrodes $(51,52)$. Also, the isolation between electrodes may be improved in the future by using nerve-growth factor to encourage nerve growth onto the electrodes. There is some controversy about the number of channels required for adequate speech perception, but it seems likely that in difficult listening situations, such as when background sounds are present, at least 16 channels are required (53-56).

Apart from problems caused by the inadequate number of effective channels in implants, there are several other reasons why the coding of spectral shape may be poorer than in a normal ear. First, there is typically a mismapping in the allocation of frequency bands to electrodes. For example, the output of a band centered at $1,000 \mathrm{~Hz}$ may be used to drive an electrode at the 2,000$\mathrm{Hz}$ place within the cochlea. It is clear that such mismapping can have severe deleterious effects in the short term (57), although implant users may adapt to it with extended experience (58). Second, in implant systems using pulsatile stimulation, the signals delivered to the implanted electrodes usually do not convey detailed temporal information relating to formant frequencies, owing to the low pulse rates used. Thus, the second of the normal coding mechanisms described above cannot operate. Third, the phase change across electrodes is not normal, so across-channel coincidence detection, the third coding mechanism described above, also cannot operate. A final problem is that there may be regions of missing neurons in the cochlea (dead regions, or "holes," in hearing) (59). An electrode producing maximum current in such a region will give rise to an audible sensation only if the current spreads to an adjacent region where there are surviving neurons. This produces a second type of mismapping and may also lead to a form of information overload in the adjacent region.

\section{CODING OF THE FREQUENCY OF SINEWAVES}

\section{Coding in the normal auditory system}

The subjective pitch of a sinewave is closely related to its frequency: the higher the frequency, the higher the pitch. For sinewaves, the frequency may be coded in the place that is excited most on the BM. This is the basis of the classic place theory of pitch $(10,60)$. However, this code is not robust; the position of the peak produced by a given frequency is level dependent $(61,62)$. For highfrequency sinewaves, the position of the peak in the vibration pattern shifts toward the base with increasing sound level (13). According to the place theory, this would be expected to lead to an increase in pitch with increasing level. Although changes in pitch with level do occur, they vary across listeners $(63,64)$ and are usually much smaller than predicted by the place theory (16).

An alternative code for frequency is neural synchrony (phase locking). As described earlier, for frequencies up to approximately $5 \mathrm{kHz}$, nerve spikes in the auditory nerve tend to be synchronized to a particular phase of the stimulating waveform. Thus, the interspike intervals fall close to integer multiples of the period of the stimulus, and this provides a potential code for frequency (and pitch).

It has also been proposed that across-place coincidence detection may be involved in the coding of frequency $(65,66)$. As described earlier, the phase of response to a sinewave of specific frequency is different at different points on the BM. The phase difference between two specific points varies with the frequency of the input. Thus, the pattern of responses in an array of across-place coincidence detectors will be unique for each stimulating frequency.

\section{Coding in implants}

In implant systems, frequency, like spectral shape, is partly coded in the relative magnitude of the electric signal across channels. A single sinewave will give rise to an output largely restricted to a single electrode. Thus, 
a form of place coding will occur. However, this code is crude because of the small number of electrodes and the limited isolation between them. For low frequencies at least, frequency may also be coded in the time pattern of the signal applied to a single electrode. Experiments with animals suggest that phase locking to electric stimuli is very precise $(25,67)$, so one might expect that frequency could be represented very precisely in the pattern of phase locking. In practice, people with cochlear implants show a rather poor ability to detect changes in the periodicity of the electric waveform applied to a single electrode, and most cannot discriminate changes at all for repetition rates above approximately $300 \mathrm{~Hz}(27,28)$.

There may be several reasons for the relatively poor ability of implantees to discriminate changes in frequency using a temporal code. First, the animal experiments measuring the precision of phase locking have typically been performed using currents such that a spike is evoked on every stimulus cycle (or for every pulse). In human implantees, such currents would lead to a very loud sound and would probably be unacceptable. For more realistic current levels, phase locking may be much less precise. Second, there is often a mismatch between temporal and place information. For example, a signal with a repetition rate of $500 \mathrm{~Hz}$ may be delivered to a place that would normally be tuned to $1,000 \mathrm{~Hz}$. The auditory system may require a correspondence between the place and temporal information to extract accurate information about frequency (68). Third, as discussed earlier, there will often be partial degeneration of the auditory nerve. This may adversely affect performance simply because there are fewer neurons carrying the temporal information. Finally, in an implantee, the phase differences across channels that would normally be produced by the traveling wave on the BM are absent. This prevents coding of frequency based on across-channel coincidence detection $(65,66)$.

It should be noted that many implant processors do not even attempt to convey information about fine time structure for frequencies above a few hundred Hertz. This feature of implant design is based on the empirical finding that implantees make little or no use of fine time structure information at high frequencies. It remains to be seen whether implantees can make more effective use of temporal information with newer stimulation strategies, such as those using very high pulse rates (69).

\section{CODING OF PERIODICITY AND PITCH OF COMPLEX TONES}

Coding in the normal auditory system

Periodic complex sounds such as those produced by musical instruments and the human voice evoke a sense of pitch. The pitch that is heard is generally very close to the pitch of the fundamental frequency of the complex sound. For example, if the sound has a repetition rate of 256 times per second, the fundamental frequency is 256 $\mathrm{Hz}$, and the pitch is close to that of a sinewave with a frequency of $256 \mathrm{~Hz}$. However, the fundamental compo- nent can be removed from the sound, and the pitch remains unaltered (70). The low pitch that is heard when the fundamental component is not present is called the pitch of the missing fundamental, residue pitch (70), virtual pitch (71), or just low pitch (72).

Several models have been proposed to account for the perception of the low pitch of complex tones. To understand how these models work, it is useful to consider a simulation of the response of the BM to a complex sound with many harmonics. Such a simulation is shown in Figure 5; the input was a series of brief pulses, repeating 200 times per second. The lower harmonics in this sound each produce a peak at a specific location on the BM. Effectively, the lower harmonics are resolved or separated on the BM. For example, the $600-\mathrm{Hz}$ harmonic produces a local peak at the place tuned to $600 \mathrm{~Hz}$. The timing of the neural spikes derived from such a place relates to the frequency of the individual harmonic rather than to the repetition rate of the complex as a whole. For example, in neurons with CFs close to $600 \mathrm{~Hz}$, the interspike intervals are close to integer multiples of 1.667

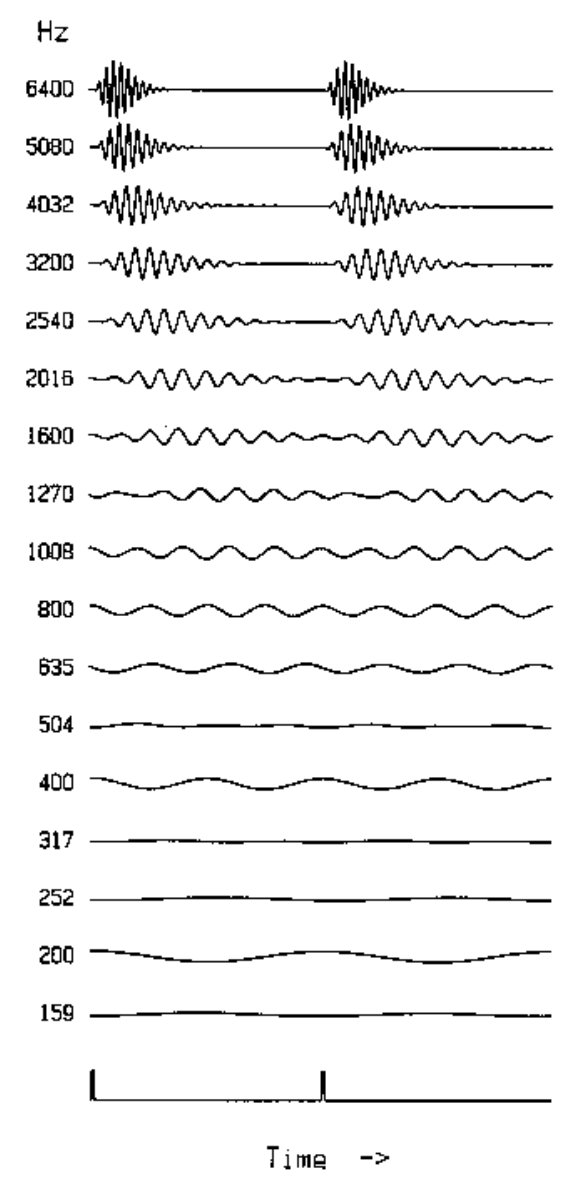

FIG. 5. Simulation of the responses on the basilar membrane to periodic impulses at a rate of 200 pulses per second. Each number on the left represents the frequency that would maximally excite a given point on the basilar membrane. The waveform that would be observed at that point, as a function of time, is plotted opposite that number. 
milliseconds. However, at places on the BM responding to the higher harmonics, the harmonics are not resolved. The excitation pattern does not show local peaks corresponding to individual harmonics. In this case, the waveform on the BM results from the interference (summation) of several harmonics and shows a periodicity the same as that of the input waveform. The timing of neural impulses derived from such a region is related to the repetition rate of the original input waveform; i.e., interspike intervals close to the repetition period will be prominent.

One class of pitch model assumes that the pitch of a complex tone is derived from neural information about the frequencies or pitches of the individual harmonics $(71,73,74)$. For these models, the lower harmonics are the most important ones in determining pitch, because these are the ones that are best resolved on the BM. Information about the frequencies of individual harmonics may be carried in the rate-versus-place profile (firing rate as a function of $\mathrm{CF}$ ), in the patterns of phase-locking at specific CFs, or in the patterns of responses of acrossplace coincidence detectors. The models assume that a central auditory mechanism computes the pitch by finding a best-fitting fundamental component. For example, if there is evidence of harmonics with frequencies close to $800,1,000$ and $1,200 \mathrm{~Hz}$, the best-fitting fundamental frequency is $200 \mathrm{~Hz}$.

The second class of model assumes that the pitch of a complex tone is related to the time intervals between nerve spikes in the auditory nerve $(73,75)$. It is assumed that pitch is derived from a place on the BM where harmonics interfere strongly, i.e., a place responding to the higher harmonics. The nerve spikes tend to be evoked at the main peaks of the complex waveform evoked at this place.

Psychoacoustic research indicates that the low pitch of a complex sound can be derived either from the low resolved harmonics (76) or from the higher unresolved harmonics $(77,78)$. This has led some authors to develop hybrid pitch models based on the use of both place and temporal information $(16,79)$; these models assume that information from low and high harmonics is combined. However, the clearest pitch is heard when low harmonics are present $(80,81)$, and the ability to detect changes in repetition rate is best when low harmonics are present $(82,83)$. Thus, it seems that a mechanism deriving pitch from the resolved harmonics is dominant.

\section{Coding in implants}

Current methods of coding used in cochlear implants do not make effective use of the most important coding mechanism for pitch in the normal auditory system, namely, the derivation of pitch from information about the frequencies of resolved harmonics. The lower harmonics in complex tones, such as those produced by the human voice, are not usually resolved by the filters in typical implant processors, except perhaps when the fundamental frequency of the input is relatively high (e.g., a child's voice). Even if they were, the implant user would be unable to extract accurate information about the fre- quencies of individual harmonics because of the limitations in place and temporal coding discussed above. Thus, pitch extraction based on the frequencies of resolved harmonics cannot operate. Coding of the periodicity of complex sounds in implants depends almost entirely on a temporal code comparable to that used in normally hearing people when a complex sound contains only high harmonics. This leads to relatively poor discrimination.

\section{BINAURAL HEARING}

Benefits of two ears for normal listeners

There are several advantages of having two normal ears. First, differences in the intensity and time of arrival of sounds at the two ears provide cues that are used to localize sound sources in space $(84,85)$. Normally hearing people can detect a change in interaural time difference as small as 10 microseconds $(16,85)$. Second, when a desired signal and a background noise come from different locations, comparison of the stimuli reaching the two ears improves the ability to detect and discriminate the signal in the noise $(86,87)$. Third, when trying to hear a sound such as speech in the presence of background noise, the speech-to-noise ratio may be much higher at one ear than at the other ear, as a result of acoustic head-shadow effects. For example, if the speech comes from the left and the noise from the right, the speech-tonoise ratio will be higher at the left ear than at the right. Under these circumstances, people are able to make use of the ear receiving the higher speech-to-noise ratio (88). Finally, even when the signals reaching the two ears are identical, the ability to discriminate or identify the signals is often slightly better than when the signals are delivered to one ear only $(89,90)$.

\section{Potential benefits of bilateral cochlear implantation}

Currently, it is common practice for cochlear implants to be implanted unilaterally. This means that all the benefits of having two ears, as described above, are lost. In recent years, a few patients have been given bilateral implants, and the results have been encouraging (91-93). It should be noted that bilateral cochlear implants are unlikely to restore to normal the ability to use interaural time differences to localize sounds and improve the detection of sounds in noise. This is because the processing of interaural time delays in the normal auditory system probably involves a system of delay lines and coincidence detectors that are tonotopically organized $(94,95)$. Essentially, the output of given place in one ear is crosscorrelated with the output from the corresponding place in the opposite ear (96); the two places have the same CF. In a person with bilateral cochlear implants, it is very unlikely that the electrode arrays would be inserted to exactly the same depth in the two ears, so there would be a misalignment across ears; the neurons excited maximally by the $n$th electrode in one ear would have different CFs from the neurons maximally excited by the $n$th electrode in the other ear. This means that the normal 
process for measuring interaural time delays will not work. Also, for bilateral implant systems using pulsatile stimulation, the timing of the pulses is not usually synchronized across ears. Again, this is likely to disrupt the processing of interaural time differences.

However, bilateral implantation is likely to give benefits from head-shadow effects; the implantee can select the ear giving the higher signal-to-background ratio at any instant. Also, bilateral implantation may help to prevent the progressive neural degeneration that would otherwise occur in a nonimplanted ear.

\section{Sound localization based on pinna cues}

Sound localization by normally hearing listeners depends partly on the use of high-frequency spectral cues, which are available even when only one ear is used. Some of the sound from a given source enters the meatus directly, but some enters the meatus after reflection from one or more of the folds in the pinna. There are also some reflections from the shoulders and the upper part of the torso. When the direct sound and the reflected sound are added together, this results in interference effects, giving a change in the spectrum of the sound reaching the eardrum. Because there are usually several reflections from the pinna, the spectrum of the sound reaching the eardrum is complex, containing multiple peaks and dips. The spectral pattern varies systematically with the direction of the sound source, and this information is used for localization, particularly in telling whether a sound is coming from in front or behind the head, and below or above the head $(85,97)$. The spectral cues provided by the pinna occur mainly at high frequencies; the frequency range above $6 \mathrm{kHz}$ is especially important.

For people with cochlear implants, spectral cues provided by the pinna are usually not available, for two reasons. First, the microphone of the implant system is usually located above and behind the ear; to pick up the location-dependent spectral changes introduced by the pinna, the microphone would need to be located in the ear canal or at its entrance. Second, cochlear implants do not typically provide the detailed spectral information at high frequencies that would be required to make use of pinna cues.

\section{STOCHASTICITY AND INDEPENDENCE ACROSS CHANNELS}

In the normal auditory nerve, the firing pattern of individual neurons is not completely predictable. The response can be characterized on average, but there is an inherent variability or randomness in the responses; the responses are said to be stochastic. The spike pattern evoked by a completely fixed stimulus will vary from one presentation to the next. In response to a sinewave, a spike will not occur for every cycle of a stimulus, although when spikes do occur, this happens at roughly the same phase of the waveform for each spike. Furthermore, the random variation in spike initiation is indepen- dent across neurons. Thus, in response to a sinewave, each neuron will show a different spike pattern.

It is not known whether independence of responses across neurons is important, but some researchers have proposed that it is $(98,99)$. In most current implant systems, electric stimulation results in highly correlated firing patterns across neurons, which is unlike what occurs in the normal auditory system. The pattern of firing across neurons in an electrically stimulated ear can be made more independent by using very high pulse rates for CIS-type strategies $(69,100)$. Another approach, used mainly with compressed-analog stimulation, is to add low-level background noise, with independent noise on each channel $(98,99)$. Further research is needed to establish the benefits of these approaches.

The stochastic responses of normal auditory neurons may well be important for the neural coding of weak signals. With electric stimulation, it may take only a small increase in current to change a neuron from not responding at all to responding on every cycle of the stimulus (or every pulse of a pulsatile stimulus). This makes it difficult to code small changes in level of a weak signal. The effect is analogous to that encountered when the attempt to make a digital recording of a weak signal, close in magnitude to the least significant bit. Either the signal is not detected at all, or it is coded very crudely as a sequence of zeros and ones. A sinewave signal would effectively be converted to a square wave. This problem is well known in audio engineering and is solved by adding a very weak noise to the desired audio signal. The noise is called dither (101). The effective dynamic range of a digital recording can be markedly increased by the use of dither (102). Almost every compact disc is recorded using dither, which is added either deliberately or inadvertently as a result of microphone noise. The beneficial effects of adding noise to the channel signals in a cochlear implant may be similar in nature; by dithering the electric signal, coding of weak signals may be enhanced, and dynamic range extended.

\section{CONCLUSIONS}

The large dynamic range of the normal ear results partly from a fast-acting compression mechanism in the cochlea. This mechanism is bypassed in cochlear implants. It is probably best not to compensate for this entirely with fast-acting compression in an implant processor. A combination of a slow-acting front-end automatic gain control with a moderate amount of fast compression in individual channels may be the best compromise.

The effective number of frequency channels provided by an implant is less than in a normal ear. This limits the precision with which information about spectral shape and time can be coded. More and better isolated channels are needed. Modiolar-hugging electrodes are a step forward. Further improvements may be obtained by encouraging nerve growth onto electrodes.

The normal ear may use across-channel coincidence detection to code sound level, spectral shape, and pitch. 
This code is not represented in any current implant because it depends on differences in phase response at different points along the basilar membrane. To implement this code requires many overlapping channels with a smoothly varying phase response across channels.

The perception of the pitch of complex tones in a normal ear depends partly on the time pattern of the higher unresolved harmonics but mainly on information derived from the lower resolved harmonics. The latter mechanism is inoperative in cochlear implants, because implants do not convey effective information about the frequencies of individual harmonics. Hence, the resolution and precision of the pitch perception of complex tones is much worse for implantees than for normally hearing people.

Two ears convey many advantages for normally hearing people, both for sound localization and for the detection and discrimination of sounds in the presence of background noise. Some but not all of these advantages may be achieved by bilateral cochlear implantation. It seems unlikely that binaural implantation will restore to normal the ability to use interaural time differences for localization and detection, but bilateral implantation is likely to improve the ability to understand speech in the presence of interfering sounds coming from other directions.

The normal auditory system uses spectral cues produced by reflection of sound from the pinna to localize sounds, and particularly to resolve up-down and frontback confusions. These cues are not conveyed by current cochlear implant systems, because the microphone is located behind rather than in the ear, and because the systems do not convey sufficient information about spectral shape at high frequencies.

The independence of neural firing across neurons in cochlear implantees may be increased by using very high pulse rates (for CIS-like strategies) or by adding lowlevel noise (for analog strategies). This may enhance the coding of low-level sounds. However, the benefits of these approaches remain to be proved.

Acknowledgments: The author thanks Michael Stone for helpful comments on an earlier version of this paper.

\section{REFERENCES}

1. Parkins CW. Compensating for hair cell loss with cochlear implants. In: Berlin CI, ed. Neurotransmission and Hearing Loss. San Diego: Singular, 1997;107-35.

2. Schuknecht HF. Pathology of the Ear. Cambridge, MA: Harvard University Press; 1974.

3. Incesulu A, Nadol JB Jr. Correlation of acoustic threshold measures and spiral ganglion cell survival in severe to profound sensorineural hearing loss: implications for cochlear implantation. Ann Otol Rhinol Laryngol 1998;107:906-11.

4. Ashmore JF. A fast motile response in guinea pig outer hair cells: the cellular basis of the cochlear amplifier. J Physiol 1987;388: 323-47.

5. Yates GK. Cochlear structure and function. In: Moore BCJ, ed. Hearing. San Diego: Academic Press, 1995;41-73.

6. Robles L, Ruggero MA. Mechanics of the mammalian cochlea. Physiol Rev 2001;81:1305-52.
7. Camalet S, Duke T, Julicher F, Prost J. Auditory sensitivity provided by self-tuned critical oscillations of hair cells. Proc Natl Acad Sci U S A 2000;97:3183-8.

8. Eguiluz VM, Ospeck M, Choe Y, Hudspeth AJ, Magnasco MO. Essential nonlinearities in hearing. Phys Rev Lett 2000;84: 5232-5.

9. Ashmore JF, Mammano F. Can you still see the cochlea for the molecules? Curr Opin Neurobiol 2001;11:449-54.

10. von Békésy G. Experiments in Hearing. New York: McGrawHill, 1960.

11. Sellick PM, Patuzzi R, Johnstone BM. Measurement of basilar membrane motion in the guinea pig using the Mössbauer technique. J Acoust Soc Am 1982;72:131-41.

12. Robles L, Ruggero MA, Rich NC. Basilar membrane mechanics at the base of the chinchilla cochlea: I. Input-output functions, tuning curves, and response phases. J Acoust Soc Am 1986;80: 1364-74.

13. Ruggero MA, Rich NC, Recio A, Narayan SS, Robles L. Basilarmembrane responses to tones at the base of the chinchilla cochlea. J Acoust Soc Am 1997;101:2151-63.

14. Stevens SS. On the psychophysical law. Psych Rev 1957;64: 153-81.

15. Hellman RP. Growth of loudness at 1000 and $3000 \mathrm{~Hz}$. J Acoust Soc Am 1976;60:672-9.

16. Moore BCJ. An Introduction to the Psychology of Hearing. 4th ed. San Diego: Academic Press, 1997.

17. Relkin EM, Doucet JR. Is loudness simply proportional to the auditory nerve spike count? J Acoust Soc Am 1997;191:2735-40.

18. Liberman MC. Auditory-nerve response from cats raised in a low-noise chamber. J Acoust Soc Am 1978;63:442-55.

19. Sachs MB, Abbas PJ. Rate versus level functions for auditorynerve fibers in cats: tone-burst stimuli. J Acoust Soc Am 1974; 56:1835-47.

20. Delgutte B. Peripheral auditory processing of speech information: implications from a physiological study of intensity discrimination. In: Schouten MEH, ed. The Psychophysics of Speech Perception. Dordrecht, The Netherlands: Nijhoff; 1987.

21. Delgutte B. Physiological models for basic auditory percepts. In: Hawkins HL, McMullen TA, Popper AN, Fay RR, eds. Auditory Computation. New York: Springer, 1996;157-220.

22. Palmer AR, Russell IJ. Phase-locking in the cochlear nerve of the guinea pig and its relation to the receptor potential of inner haircells. Hear Res 1986;24:1-15.

23. Young ED, Sachs MB. Representation of steady-state vowels in the temporal aspects of the discharge patterns of populations of auditory-nerve fibers. J Acoust Soc Am 1979;66:1381-403.

24. Heinz MG, Colburn HS, Carney LH. Rate and timing cues associated with the cochlear amplifier: level discrimination based on monaural cross-frequency coincidence detection. J Acoust Soc Am 2001;110:2065-84.

25. Kiang NY, Moxon EC. Physiological considerations in artificial stimulation of the inner ear. Ann Otol Rhinol Laryngol 1972;81: 714-30.

26. Javel E, Viemeister NF. Stochastic properties of cat auditory nerve responses to electric and acoustic stimuli and application to intensity discrimination. J Acoust Soc Am 2000;107:908-21.

27. Bilger RC. Electrical stimulation of the auditory nerve and auditory prostheses: a review of the literature. Ann Otol Rhinol Laryngol 1977;86(Suppl 38):11-20.

28. Fourcin AJ, Rosen SM, Moore BCJ, et al. External electrical stimulation of the cochlea: clinical, psychophysical, speechperceptual and histological findings. Br J Audiol 1979;13:85-107.

29. Pearsons KS, Bennett RL, Fidell S. Speech Levels in Various Environments. Report No. 3281. Cambridge, MA: Bolt, Beranek and Newman; 1976.

30. Killion MC. Hearing aids: past, present and future: moving toward normal conversations in noise. Br J Audiol 1997;31:141-8.

31. Steinberg JC, Gardner MB. The dependency of hearing impairment on sound intensity. J Acoust Soc Am 1937;9:11-23.

32. Villchur E. Signal processing to improve speech intelligibility in perceptive deafness. J Acoust Soc Am 1973;53:1646-57.

33. Moore BCJ. Cochlear Hearing Loss. London: Whurr, 1998. 
34. Stone MA, Moore BCJ, Alcántara JI, Glasberg BR. Comparison of different forms of compression using wearable digital hearing aids. J Acoust Soc Am 1999;106:3603-19.

35. Moore BCJ, Glasberg BR. A comparison of four methods of implementing automatic gain control (AGC) in hearing aids. $\mathrm{Br} J$ Audiol 1988;22:93-104.

36. Moore BCJ, Glasberg BR, Stone MA. Optimization of a slowacting automatic gain control system for use in hearing aids. $\mathrm{Br} \mathrm{J}$ Audiol 1991;25:171-82.

37. Stöbich B, Zierhofer CM, Hochmair ES. Influence of automatic gain control parameter settings on speech understanding of cochlear implant users employing the continuous interleaved sampling strategy. Ear Hear 1999;20:104-16.

38. Recio A, Rich NC, Narayan SS, Ruggero MA. Basilar-membrane responses to clicks at the base of the chinchilla cochlea. J Acoust Soc Am 1998;103:1972-89.

39. Verschuure J, Prinsen TT, Dreschler WA. The effect of syllabic compression and frequency shaping on speech intelligibility in hearing impaired people. Ear Hear 1994;15:13-21.

40. van Buuren RA, Festen J, Houtgast T. Compression and expansion of the temporal envelope: evaluation of speech intelligibility and sound quality. J Acoust Soc Am 1999;105:2903-13.

41. Plomp R. The role of modulation in hearing. In: Klinke R, Hartmann R, eds. Hearing: Physiological Bases and Psychophysics. Berlin: Springer, 1983;270-6.

42. Drullman R, Festen JM, Plomp R. Effect of temporal envelope smearing on speech reception. J Acoust Soc Am 1994;95: 1053-64.

43. Stone MA, Moore BCJ. Syllabic compression: effective compression ratios for signals modulated at different rates. $\mathrm{Br} J$ Audiol 1992;26:351-61

44. Noordhoek IM, Drullman R. Effect of reducing temporal intensity modulations on sentence intelligibility. J Acoust Soc Am 1997; 101:498-502.

45. Loizou PC, Dorman M, Fitzke J. The effect of reduced dynamic range on speech understanding: implications for patients with cochlear implants. Ear Hear 2000;21:25-31.

46. Bregman AS. Auditory Scene Analysis: The Perceptual Organization of Sound. Cambridge, MA: Bradford Books, MIT Press, 1990.

47. Glasberg BR, Moore BCJ. Derivation of auditory filter shapes from notched-noise data. Hear Res 1990;47:103-38.

48. ANSI. ANSI S3.5-1997, Methods for the calculation of the speech intelligibility index. New York: American National Standards Institute, 1997.

49. Zwicker E. Masking and psychologic excitation as consequences of the ear's frequency analysis. In: Plomp R, Smoorenburg GF, eds. Frequency Analysis and Periodicity Detection in Hearing. Leiden: Sijthoff; 1970;376-94.

50. Sachs MB, Young ED. Effects of nonlinearities on speech encoding in the auditory nerve. J Acoust Soc Am 1980;68:858-75.

51. Cohen LT, Saunders E, Clark GM. Psychophysics of a prototype peri-modiolar cochlear implant electrode array. Hear Res 2001; 155:63-81.

52. Zwolan T, Kileny PR, Smith S, Mills D, Koch D, Osberger MJ. Adult cochlear implant patient performance with evolving electrode technology. Otol Neurotol 2001;22:844-9.

53. Shannon RV, Zeng F-G, Kamath V, Wygonski J, Ekelid M. Speech recognition with primarily temporal cues. Science 1995 ; 270:303-4.

54. Loizou PC, Dorman M, Tu Z. On the number of channels needed to understand speech. J Acoust Soc Am 1999;106:2097-103.

55. Dorman MF, Loizou PC, Kemp LL, Kirk KI. Word recognition by children listening to speech processed into a small number of channels: data from normal-hearing children and children with cochlear implants. Ear Hear 2000;21:590-6.

56. Friesen LM, Shannon RV, Baskent D, Wang X. Speech recognition in noise as a function of the number of spectral channels: comparison of acoustic hearing and cochlear implants. J Acoust Soc Am 2001;110:1150-63.

57. Shannon RV, Zeng F-G, Wygonski J. Speech recognition with altered spectral distribution of envelope cues. J Acoust Soc Am 1998;104:2467-76.

58. Rosen S, Faulkner A, Wilkinson L. Adaptation by normal listeners to upward spectral shifts of speech: implications for cochlear implants. J Acoust Soc Am 1999;106:3629-36.

59. Moore BCJ. Dead regions in the cochlea: diagnosis, perceptual consequences, and implications for the fitting of hearing aids. Trends Amplif 2001;5:1-34.

60. Helmholtz HLF. Die Lehre von den Tonempfindungen als physiologische Grundlage für die Theorie der Musik. 1st ed. Braunschweig: F. Vieweg, 1863.

61. Zwislocki JJ, Nguyen N. Place code for pitch: a necessary revision. Acta Otolaryngol 1999;119:140-5.

62. Moore BCJ, Alcántara JI, Glasberg BR. Behavioural measurement of level-dependent shifts in the vibration pattern on the basilar membrane. Hear Res 2002;163:101-10.

63. Terhardt E. Pitch of pure tones: its relation to intensity. In: Zwicker E, Terhardt E, eds. Facts and Models in Hearing. Berlin: Springer, 1974;350-7.

64. Verschuure J, van Meeteren AA. The effect of intensity on pitch. Acustica 1975;32:33-44.

65. Loeb GE, White MW, Merzenich MM. Spatial cross correlation: a proposed mechanism for acoustic pitch perception. Biol Cybern 1983;47:149-63.

66. Shamma S, Klein D. The case of the missing pitch templates: how harmonic templates emerge in the early auditory system. J Acoust Soc Am 2000;107:2631-44.

67. Javel E, Shepherd RK. Electrical stimulation of the auditory nerve: III. Response initiation sites and temporal fine structure. Hear Res 2000;140:45-76.

68. Evans EF. Place and time coding of frequency in the peripheral auditory system: some physiological pros and cons. Audiology 1978; 17:369-420.

69. Matsuoka AJ, Rubinstein JT, Abbas PJ, Miller CA. The effects of interpulse interval on stochastic properties of electrical stimulation: models and measurements. IEEE Trans Biomed Eng 2001; 48:416-24.

70. Schouten JF. The residue and the mechanism of hearing. Proceedings of the Koninklijke Akademie van Wetenschap 1940;43: 991-9.

71. Terhardt E. Pitch, consonance, and harmony. J Acoust Soc Am 1974;55:1061-9.

72. Plomp R. Aspects of Tone Sensation. London: Academic Press, 1976.

73. de Boer E. On the "residue" in hearing. Amsterdam: University of Amsterdam, 1956 (dissertation).

74. Goldstein JL. An optimum processor theory for the central formation of the pitch of complex tones. J Acoust Soc Am 1973;54: 1496-516.

75. Schouten JF, Ritsma RJ, Cardozo BL. Pitch of the residue. $J$ Acoust Soc Am 1962;34:1418-24.

76. Houtsma AJM, Goldstein JL. The central origin of the pitch of pure tones: evidence from musical interval recognition. J Acoust Soc Am 1972;51:520-9.

77. Moore BCJ, Rosen SM. Tune recognition with reduced pitch and interval information. $Q J$ Exp Psychol 1979;31:229-40.

78. Houtsma AJM, Smurzynski J. Pitch identification and discrimination for complex tones with many harmonics. J Acoust Soc Am 1990;87:304-10

79. Meddis R, Hewitt M. Virtual pitch and phase sensitivity of a computer model of the auditory periphery: I. Pitch identification. J Acoust Soc Am 1991;89:2866-82.

80. Ritsma RJ. Frequencies dominant in the perception of the pitch of complex sounds. J Acoust Soc Am 1967;42:191-8.

81. Plomp R. Pitch of complex tones. J Acoust Soc Am 1967;41: $1526-33$.

82. Moore BCJ, Glasberg BR. Effects of the relative phase of the components on the pitch discrimination of complex tones by subjects with unilateral and bilateral cochlear impairments. In: Duifhuis H, Wit H, Horst J, eds. Basic Issues in Hearing. London: Academic Press, 1988;421-30.

83. Moore BCJ, Peters RW. Pitch discrimination and phase sensitiv- 
ity in young and elderly subjects and its relationship to frequency selectivity. J Acoust Soc Am 1992;91:2881-93.

84. Rayleigh L. On our perception of sound direction. Phil Mag 1907;13:214-32.

85. Blauert J. Spatial Hearing: The Psychophysics of Human Sound Localization. Cambridge, MA: MIT Press, 1997.

86. Hirsh IJ. Influence of interaural phase on interaural summation and inhibition. J Acoust Soc Am 1948;20:536-44.

87. Levitt H, Rabiner LR. Binaural release from masking for speech and gain in intelligibility. J Acoust Soc Am 1967;42:601-8.

88. Bronkhorst AW, Plomp R. The effect of head-induced interaural time and level differences on speech intelligibility in noise. $J$ Acoust Soc Am 1988;83:1508-16.

89. Laurence RF, Moore BCJ, Glasberg BR. A comparison of behind-the-ear high-fidelity linear aids and two-channel compression hearing aids in the laboratory and in everyday life. $\mathrm{Br} J$ Audiol 1983;17:31-48.

90. Davis A, Haggard M, Bell I. Magnitude of diotic summation in speech-in-noise tasks: performance region and appropriate baseline. Br J Audiol 1990;24:11-6.

91. van Hoesel RJM, Clark GM. Psychophysical studies with two binaural cochlear implant subjects. J Acoust Soc Am 1997;102: 495-507.

92. Gantz BJ, Tyler RS, Rubinstein JT, et al. Binaural cochlear implants placed during the same operation. Otol Neurotol 2002;23: 169-80.
93. Tyler RS, Gantz BJ, Rubinstein JT, et al. Three-month results with bilateral cochlear implants. Ear Hear 2002;23:80S-9S.

94. Jeffress LA. A place theory of sound localization. J Comp Physiol Psychol 1948;41:35-9.

95. Palmer AR. Neural signal processing. In: Moore BCJ, ed. Hearing. San Diego: Academic Press, 1995;75-121.

96. Stern RM, Trahiotis C. Models of binaural interaction. In: Moore BCJ, ed. Hearing. San Diego: Academic Press, 1995;347-86.

97. Wightman FL, Kistler DJ. Headphone simulation of free field listening: I. Stimulus synthesis. J Acoust Soc Am 1989;85: 858-67.

98. Morse RP, Evans EF. Additive noise can enhance temporal coding in a computational model of analogue cochlear implant stimulation. Hear Res 1999;133:107-19.

99. Zeng FG, Fu QJ, Morse R. Human hearing enhanced by noise. Brain Res 2000;869:251-5.

100. Rubinstein JT, Wilson BS, Finley CC, Abbas PJ. Pseudospontaneous activity: stochastic independence of auditory nerve fibers with electrical stimulation. Hear Res 1999;127:108-18.

101. Stuart JR, Wilson RJ. Dynamic range enhancement using noiseshaped dither applied to signals with and without preemphasis. $J$ Audio Eng Soc 1994;42:400.

102. Vanderkooy J, Lipshitz SP. Resolution below the least significant bit in digital systems with dither. J Audio Eng Soc 1984;32: 106-13. 